\title{
Utilidad de las respuestas rápidas de estrés para evaluar la evolución del tratamiento en un canino con fobia social
}

\author{
Cainzos, R.P. ${ }^{1,2}$; Koscinczuk, P.,2; Rossner, M.V. ${ }^{1,2}$; \\ Alabarcez, M.N.'; Maurenzig, N.D. ${ }^{1}$ \\ ${ }^{1}$ Cátedra de Patología Médica, ${ }^{2}$ Cátedra de Bienestar Animal, Facultad de Ciencias. Veterinarias, Universidad \\ Nacional del Nordeste, Sargento Cabral 2139, Corrientes (3400), Argentina. Tel/Fax. 0379-4425753. \\ Email: romicainzos@hotmail.com.
}

\begin{abstract}
Resumen
Cainzos, R.P.; Koscinczuk, P.; Rossner, M.V.; Alabarcez, M.N.; Maurenzig, N.D.: Utilidad de las respuestas rápidas de estrés para evaluar la evolución del tratamiento en un canino con fobia social. Rev. vet. 23: 1, 49-54, 2012. El miedo es un estado emocional aversivo ante una amenaza real o percibida como peligro externo. Esta emoción produce respuestas autonómicas que alteran la homeostasis del individuo. Cuando esta situación se prolonga en el tiempo, el estado de salud se afecta. El objetivo de este trabajo fue relacionar el reflejo pupilar y las frecuencia cardíaca y respiratoria con la producción de saliva, concentración de cortisol y respuestas conductuales, para evaluar la evolución del tratamiento con paroxetina $(1 \mathrm{mg} / \mathrm{kg}$ cada 12 horas) y terapia conductual en una perra boxer con fobia social, en el primer mes post adopción. Ambas frecuencias se mantuvieron normales durante toda la experiencia (FC 76 a 98 lpm y FR 18 a $40 \mathrm{mrm}$ ). Sin embargo, en el día 1 la frecuencia cardíaca fue más alta que en los días 15 y $30(\mathrm{p}<0,05)$. Con respecto a la dilatación pupilar, en el día uno se observó midriasis y a partir del día 15 el reflejo pupilar fue normal. Si bien la producción de saliva fue más abundante en el primer día que en los días 15 y $30(p<0,05)$, las concentraciones de cortisol durante todo el periodo no arrojaron diferencias significativas, presentando el valor absoluto más alto el día $15(1,22 \mu \mathrm{g} / \mathrm{dl})$. Tanto las variables para los comportamientos de mantenimiento como para los comportamientos sociales fueron significativamente diferentes a lo largo de la experiencia $(\mathrm{p}<0,001)$. Por otra parte, no se presentaron efectos adversos atribuibles a la paroxetina y la paciente recuperó el apetito, entrando en celo a los dos meses después. Los parámetros clínicos asociados al sistema nervioso autónomo resultan de interés práctico para evaluar la progresión del tratamiento del paciente canino con fobia.
\end{abstract}

Palabras clave: perro, miedo, frecuencias cardíaca y respiratoria, cortisol salival.

\begin{abstract}
Cainzos, R.P.; Koscinczuk, P.; Rossner, M.V.; Alabarcez, M.N.; Maurenzig, N.D.: Use of the stress fast response for the evaluation of a social phobia treatment in a dog. Rev. vet. 23: 1, 49-54, 2012. Fear is an aversive state emotion to a real or perceived threat external situation. This emotion induces autonomic responses that alter homeostasis. When this situation is prolonged in time, health is affected. Aim of this work was to establish a relationship among routine clinical parameters -such as hearth and breath rates, pupil reflect with salivary production, salivary cortisol and behaviour response- for the evaluation of treatment evolution with paroxetin ( $1 \mathrm{mg} / \mathrm{kg}$ every 12 hours) associated to a behavioural therapy in a boxer bitch with social phobia, during the first month after adoption. Both heart and breath rates were between the normal range for the species during all the experiment (76 to $98 \mathrm{bpm}$ and 18 to $40 \mathrm{bpm}$ respectively. Regarding pupil dilation, on the first day the patient had mydriasis, but since day 15 she had myosis. Saliva production was more abundant on the first day, compared to days 15 and $30(p<0.05)$; salivary cortisol did not show significant differences, with the highest absolute value on day $15(1.22 \mu \mathrm{g} / \mathrm{ml})$. Variables of both maintenance and social behaviours were significatively different during the entire assay $(\mathrm{p}<0.001)$. On the other hand, there was no adverse effect associated to paroxetin and the patient recovered the appetite, presenting heat during the second month. Clinical parameters associated with the autonomic nervous system result of practical interest to evaluate the treatment evolution of a phobic patient.
\end{abstract}

Key words: dog, fear, heart and respiratory rates, salivary cortisol. 


\section{INTRODUCCIÓN}

El miedo es la aprehensión a un estímulo, objeto o evento ${ }^{3}$ considerado potencialmente peligroso ${ }^{9}$. Es una respuesta emocional que sirve como expresión de un comportamiento defensivo en situaciones de peligro. Se lo considera altamente adaptativo y esencial para la supervivencia de las especies ${ }^{3}$. Las estrategias conductuales que los animales pueden adoptar son diversas y van desde paralizarse (autoapaciguamiento) y la autodistracción, hasta escapar o pelear ${ }^{10,15}$. El comportamiento manifestado va a depender no solo del repertorio natural de la especie, fijado genéticamente, sino también de la experiencia del individuo adquirida mediante el aprendizaje ${ }^{9,10,15}$.

En todos los casos la respuesta conductual de miedo se acompaña por la activación del sistema nervioso autónomo con taquicardia/bradicardia, taquipnea, boca seca, midriasis o miosis ${ }^{4,14}$. Tal sistema se relaciona estrechamente con estructuras neuroendocrinas, particularmente con el hipotálamo, que se constituye en una pequeña central integradora ${ }^{13}$ recibiendo, también, los impulsos nerviosos de la amígdala. En la interpretación del peligro participan tanto la corteza prefrontal como la órbitofrontal a partir de la evaluación de los estímulos percibidos por los órganos de los sentidos. Ambas cortezas se articulan con la amígdala enviando y recibiendo desde allí señales nerviosas. Por otra parte es la misma corteza prefrontal que, a través del circuito tálamo, hipotálamo y amígdala, puede inhibir las respuestas endocrinas y motoras producidas en estas dos últimas. Esta labor inhibitoria del comportamiento de miedo se lleva a cabo por mediadores químicos como el GABA y los opiáceos ${ }^{12}$. La complejidad del sistema neuroendocrino y los numerosos mediadores que se ponen en juego hacen que los individuos perciban de diferente manera estímulos semejantes.

Mientras que la característica principal del miedo es el comportamiento direccional, relacionado con la localización del estímulo, la fobia es un proceso repentino, excesivo y acompañado por un profundo miedo, desencadenado por un estímulo o grupo de estímulos específicos presentes en el ambiente pero sin peligro real aparente ${ }^{3,10}$. En algunos casos puede desencadenarse sin el estímulo iniciador específico. El desarrollo de la fobia atraviesa tres estadios sucesivos, que llevan a una degradación progresiva de la homeostasis sensorial del animal, terminando con la aparición de un estadio ansioso. Cuando se llega a este estadio final se observa un miedo casi permanente, con predomino de las manifestaciones neurovegetativas, temblores y tentativas de huida. Los estímulos desencadenantes se tornan entonces muy numerosos y la anticipación emocional es muy marcada. La aparición del estadio ansioso muestra una degradación de la capacidad adaptativa del animal, con desorganización de los autocontroles y pérdida de adaptabilidad a cualquier variación del entorno ${ }^{3}$.

El tratamiento de pacientes con miedo o fobia requiere de una combinación de terapia con drogas y también de tipo conductual. Mientras que la terapia medicamentosa modifica el perfil neuroquímico del cerebro ${ }^{11}$, la terapia conductual apunta a modificaciones estables del comportamiento perdurables en el tiempo. Para lograr modificar el comportamiento se recomienda aplicar tres procesos de aprendizaje: habituación, desensibilización y condicionamiento inverso ${ }^{3,10}$; el objetivo principal es remplazar la respuesta fóbica del paciente por una que sea calmada y aceptable, cada vez que sea expuesto a estímulos desencadenantes ${ }^{10}$.

Se ha descrito que el estado psicológico que más se distingue en los perros confinados es el de miedo en cualquiera de sus categorías: miedo a los extraños, a las escaleras, al tacto ${ }^{8}$. Reconocer estas señales de miedo permitiría tomar medidas tendientes a mejorar la salud de los individuos y a minimizar el impacto que esta emoción puede ejercer sobre el bienestar de los animales, evitando que estas señales evolucionen a una patología más compleja, como la fobia.

El objetivo de este trabajo fue evaluar la evolución de los parámetros clínicos de rutina: reflejo pupilar y frecuencias cardíaca y respiratoria, asociándolos a la producción de saliva, cortisol y respuestas conductuales durante una terapia médico-conductual de un caso de fobia en una perra bóxer.

\section{MATERIAL Y MÉTODOS}

Paciente. Canino hembra, boxer de 2 años de edad y $18 \mathrm{~kg}$ de peso inicial, procedente de un criadero, con anorexia asociada a fobia luego de ser adoptada por una familia. El criterio de selección se basó en los signos clínicos que presentaba la paciente al momento de comenzar el estudio: trataba de huir o esconderse y luego quedaba estática, con inhibición de otros comportamientos, llegando a la anorexia, cola entre las patas, grupa descendida y orejas retraídas hacia caudal.

Parámetros clínicos. Para evaluar las respuestas rápidas de estrés se registraron: frecuencia cardíaca (latidos por minuto, $1 \mathrm{pm}$ ) y respiratoria (movimientos respiratorios por minuto, mrm). La dilatación de la pupila se registró por observación directa durante $10 \mathrm{~s}$ en una habitación con luz artificial indirecta con intensidad suficiente como para permitir la lectura. Se registró como ausencia o presencia de dilatación (presencia es sinónimo de midriasis). La recolección de estos datos se realizó los días 1,15 y 30 , en tres oportunidades cada día (7, 14 y 19 h), a los efectos de registrar las variaciones asociadas al ritmo circadiano.

Producción de saliva y cortisol. Los días 1, 15 y 30, en tres oportunidades durante el día (7, 14 y 19 h), junto con la obtención de los parámetros clínicos, se recolectó una muestra de saliva mediante hilo dental de algodón colocado en la boca durante aproximadamente 1 minuto $(\mathrm{ml} / \mathrm{min})$. Antes de esta maniobra el propietario se aseguró que la perra no hubiera ingerido alimentos ni masticado objetos media hora antes. El material recolectado se colocó en un tubo Eppendorf. Antes de ser refrigerada a $-4^{\circ} \mathrm{C}$, se midió el volumen obtenido 
con una jeringa descartable. La determinación de la concentración de cortisol salival se realizó mediante método inmunoenzimático. El test usa $25 \mu \mathrm{l}$ de saliva (para cada determinación individual) y tiene un rango de sensibilidad entre 0,007 y $1,8 \mu \mathrm{g} / \mathrm{dl}$.

Observación del comportamiento. Diariamente se realizó observación focal del animal durante $10 \mathrm{minu}-$ tos, después de cada toma de muestra de saliva. Se tuvieron en cuenta las siguientes variables conductuales: posición de las orejas, movimiento de la cola, acto de vocalización (llanto o ladrido), defecación, micción y alimentación. Además, dentro de los comportamientos sociales se evaluaron: respuesta al nombre, acercamiento voluntario a los individuos del grupo familiar, relación con otros perros y personas, conducta de juego durante el paseo, con objetos y con las personas conocidas y no conocidas.

Terapia. Para la terapia médica se utilizó paroxetina en dosis de $1 \mathrm{mg} / \mathrm{kg}$ cada 12 horas (Tiarix ${ }^{\circledR}$, comprimidos de $20 \mathrm{mg}$ ). Esta droga fue seleccionada por ser un inhibidor selectivo de la recaptación de serotonina. En cuanto a la terapia conductual aplicada conjuntamente al tratamiento médico, se comenzó con habituación y condicionamiento inverso. Como primera medida, se favoreció una respuesta calmada y relajada por parte del animal, evitando la presencia del estímulo iniciador de la fobia y los ruidos, propiciándole un lugar confiable donde esconderse o escapar, ignorando al paciente cada vez que se presentaron los signos autonómicos asociados a la patología, a los efectos de lograr la extinción de la respuesta fóbica. Se delimitó un área de descanso usando una manta marcada con sus olores.

Durante todo el ensayo se instruyó a los propietarios de no invadir ese espacio ni con premios ni con castigos. Durante este período el único ejercicio que se realizó fue el de responder a la orden de sentado. Las sesiones de entrenamiento se realizaron dos veces por día, prolongándose entre 10 y 20 minutos y cada vez que el animal respondía a la orden de sentado o quieto se le acariciaba. Paulatinamente, los propietarios fueron añadiendo algunas distracciones mientras realizaban este ejercicio, como aplaudir, hablar, levantar la mano, dar unos pasos lejos del animal y luego regresar.

Cuando la perra comenzó a acercarse voluntariamente a los miembros de la familia se prosiguió con una terapia de desensibilización, que es un proceso de modificación del comportamiento cuyo objetivo es suprimir la sensibilidad a un estímulo, exponiendo al sujeto a dicho estímulo de forma repetitiva y a intensidad creciente. En este caso se trató de controlar los diferentes estímulos: sonoros, visuales y táctiles (golpe de puertas, utensilios de cocina, ruidos de autos, presencia humana, otros animales).

Análisis estadístico. Se realizó un análisis estadístico paramétrico para las variables frecuencia cardíaca, respiratoria, producción de saliva y niveles salivales de cortisol, en tanto que para las variables conductuales se aplicó el test de Kruskal Wallis, con un nivel de significancia del 5\% (InfoStat, 2008).
Tabla 1. Obtención de saliva ( $\mathrm{ml} / \mathrm{min})$.

\begin{tabular}{cccc}
\hline día & $\overline{\mathrm{x}}$ & $\mathrm{DE}$ & rango \\
\hline 1 & 0,58 & 0,08 & $0,50-0,70$ \\
15 & 0,52 & 0,17 & $0,30-0,80$ \\
30 & 0,37 & 0,06 & $0,30-0,40$ \\
\hline
\end{tabular}

X media aritmética, DE: desvío estándar.

Las diferencias entre promedios fueron no significativas.

\section{RESULTADOS Y DISCUSIÓN}

En la paciente, la frecuencia cardíaca demostró ser útil para evaluar el progreso del tratamiento de la fobia. De hecho, se ha demostrado que también es eficaz para valorar la actividad autonómica en situaciones de estrés bajo condiciones de alojamiento deficiente y para evaluar las condiciones patológicas asociadas a desórdenes conductuales ${ }^{14}$. Es dable destacar que la respuesta del reflejo pupilar, de fácil aplicación en la clínica diaria, se asoció significativamente con el progreso del tratamiento al igual que los parámetros conductuales seleccionados del etograma.

Tanto la frecuencia cardíaca como la respiratoria se mantuvieron dentro del rango normal para la especie durante toda la experiencia (76 a 98 lpm y 18 a 40 mrm). Sin embargo, es de destacar que en el día 1 la frecuencia cardíaca fue más alta que en los días $15 \mathrm{y}$ $30(\mathrm{p}<0,05)$. Tal aumento coincide con lo observado en otro estudio de dos grupos de perros collies, los cuales tenían miedo a los disparos y a pisos desconocidos y en donde el aumento de la frecuencia cardíaca se asoció a un aumento de la presión sanguínea y del hematocrito ${ }^{5}$ . Cuando el individuo percibe una situación que puede ser potencialmente peligrosa, la amígdala, que participa en la integración de los circuitos asociados con miedo, controla las respuestas de dos maneras: directamente a través del núcleo dorsal del vago e indirectamente a través del hipotálamo lateral ${ }^{3}$, produciendo respuestas autonómicas asociadas a un aumento de la actividad simpática tal como se constató en la paciente.

Aunque inicialmente la perra presentó midriasis y abundante salivación ( $\overline{\mathrm{x}}=0,58 \mathrm{ml} / \mathrm{min})$, a partir del día 15 el reflejo pupilar fue normal, y la producción de saliva fue menor (Tabla 1). En los pacientes con miedo o fobia, el tono parasimpático sobrepasa al simpático manifestándose los signos clínicos de midriasis y aumento de producción saliva ${ }^{3}$. Como es sabido, tanto las descargas adrenérgicas como el aumento del tono vagal pueden generarse por cambios ambientales e influir sobre la función cardíaca ${ }^{1}$, por este motivo se recomienda que los estudios no invasivos deberían realizarse en los animales en reposo siempre después de un proceso de aclimatación ${ }^{14}$. Resulta interesante destacar que aquellos perros que poseen un alto tono vagal podrían mostrar menos signos de ansiedad y miedo durante los procesos de aprendizaje que acompañan a la terapia conductual $^{1}$, estando más activos y capaces de aprender y modificar su comportamiento. 
Al mismo tiempo que se produce una respuesta simpática, el hipotálamo controla la liberación de corticoesteroides a partir de la corteza adrenal ${ }^{3}$. Mientras que algunos autores encontraron correlación entre miedo a los disparos con aumento de la frecuencia cardíaca y aumento significativo de los niveles plasmáticos de cortisol en perros collies ${ }^{5}$, en el presente ensayo no se hallaron diferencias significativas en la concentración salival de cortisol durante todo el periodo de estudio (Tabla 2), no obstante el valor absoluto más alto se registró el día $15(1,22 \mu \mathrm{g} / \mathrm{dl})$ y el más bajo el día $30(0,04 \mu \mathrm{g} / \mathrm{dl})$. Esto podría ser el resultado de una activación del eje a partir de la segunda semana, ya que los perros adoptan dos estrategias para adaptarse: muestran comportamiento de miedo con menor habilidad para aprender tareas, lo que refleja un tipo de respuesta reactiva ${ }^{2}$ (lo que pudo haber sucedido durante la primer semana), o tienen una activación del eje con una mejor habilidad para responder a las nuevas tareas, lo que implica un estilo proactivo ${ }^{14}$.

Se debe recordar que el control hipotalámico de la liberación de corticosteroides a partir de la corteza adrenal no solo se produce ante reacciones de miedo, sino también ante numerosas respuestas emocionales, incluyendo aquellas de naturaleza positiva ${ }^{3}$, es por eso que una interpretación de valores absolutos sin tener en cuenta las variaciones conductuales no es suficiente para una interpretación de la actividad del eje. Si bien algunos autores consideran que la medición de cortisol entre las 14 y 16 h es estable ${ }^{6}$, cabría considerar las variaciones asociadas a la actividad y el ambiente ${ }^{6,7}$.

Si bien no se encontró una correlación entre la concentración de cortisol en la saliva y las variables del comportamiento evaluadas (Tablas 3 y 4) como la hallada por otros autores ${ }^{1}$, sí fueron verificadas diferencias significativas entre la primera, la segunda y la tercer semana post-adopción, tanto para los comportamientos de mantenimiento, como para los diferentes comportamientos sociales $(\mathrm{p}<0,001)$. En el primer día se registró que la perra olfateó algunos sitios de la casa, no movía la cola y sus orejas estaban en posición rígida la mayor parte del tiempo (Figura 1). Esta posición corporal se relaciona con una conducta de apaciguamiento ${ }^{8}$. Al ofrecerle alimento de preparación casera lo aceptó, se escondió debajo de la mesa y ante todo ruido fuerte se refugiaba en el espacio designado para su descanso, señalado por un objeto previamente marcado con sus olores. Esta respuesta de "huida" responde a la necesidad del animal de evitar una confrontación con la situación que disparó la conducta de miedo ${ }^{9}$.

Al aplicar los diferentes procesos de aprendizaje se instruyó cuidadosamente a los propietarios acerca de los eventos que podían ser considerados como peligrosos para el paciente. Si bien las personas no tienen dificultad para identificar el miedo, muchas veces no son capaces de reconocer las pistas ambientales que afectan al individuo. Al iniciarse el proceso de adopción, el ambiente no es familiar y es muy factible que provoque miedo; el animal no reconoce a las personas como parte de su grupo. Por eso es importante definir el umbral
Tabla 2. Cortisol salival ( $\mu \mathrm{l} / \mathrm{dl})$.

\begin{tabular}{cccc}
\hline día & $\overline{\mathrm{x}}$ & DE & rango \\
\hline 1 & 0,77 & 0,24 & $0,53-1,14$ \\
15 & 0,86 & 0,19 & $0,69-1,22$ \\
30 & 0,31 & 0,46 & $0,04-0,83$ \\
\hline
\end{tabular}

$\overline{\mathrm{X}}$ media aritmética, DE: desvío estándar. Las diferencias entre promedios fueron no significativas.

espacial alocéntrico, es decir definir la frontera geográfica que el individuo considerará como su territorio, en este caso representado por una manta con sus olores colocada debajo de una mesa. El umbral espacial egocéntrico en el perro (en el ser humano se refiere como espacio personal) está representado como invasión del espacio de fuga y la invasión de su superficie corporal. Dentro de la invasión al espacio corporal ("tocarlos", por ejemplo), se reconoce que toleran mejor el contacto en el torso que en la cara ${ }^{9}$. En este caso en particular, los propietarios evitaron el contacto con su mascota.

El día 15 se observó que la perra comenzó a mover la cola y a establecer contacto visual con todos los miembros de la familia, acercándose voluntariamente a las personas que más a menudo visitaban el hogar, colocaba las orejas hacia atrás, respondía al nombre y comía

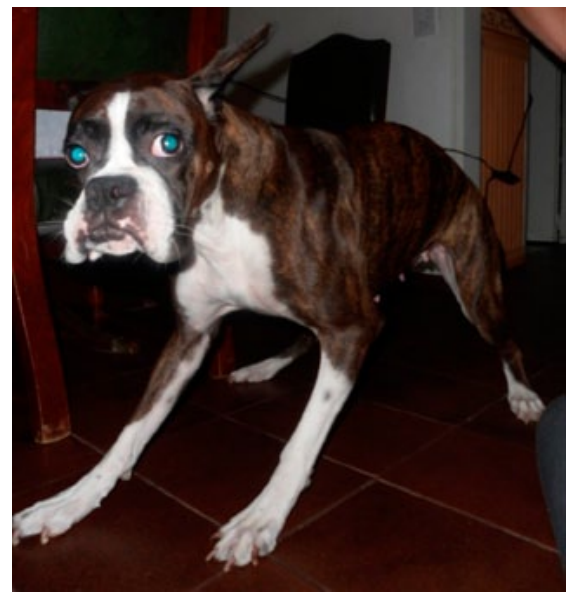

Figura 1. Posición estática, orejas rígidas, cuello en tensión, midriasis.

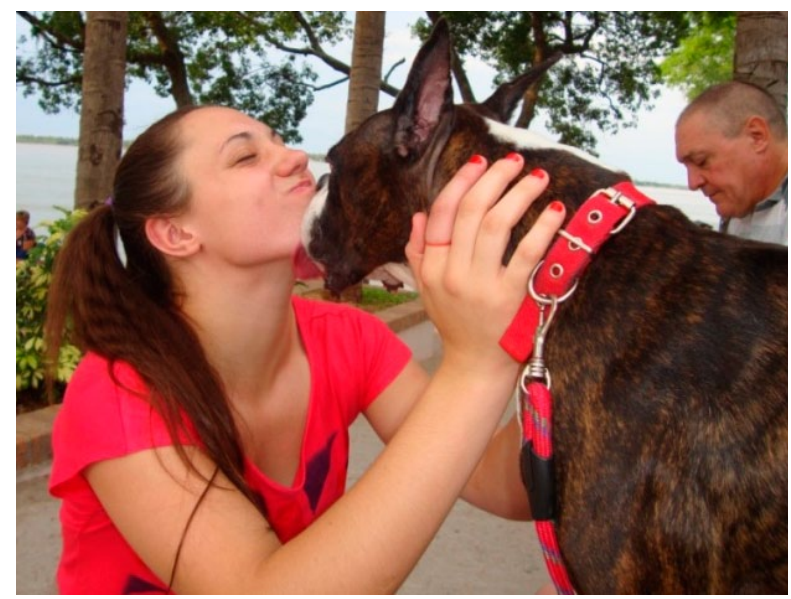

Figura 2. Iniciativa de juego con una persona conocida durante el paseo. 
Tabla 3. Comportamientos de mantenimiento registrados en las sesiones experimentales. Componentes, variables y scores definidos.

\begin{tabular}{|c|c|c|c|}
\hline $\begin{array}{l}\text { categoría del } \\
\text { comportamiento }\end{array}$ & comportamiento & descripción de las variables & score \\
\hline \multirow{2}{*}{$\begin{array}{l}\text { alimentación } \\
\text { (dos veces diarias) }\end{array}$} & comida casera & $\begin{array}{l}\text { no la come } \\
\text { se acerca, olfatea, come un poco } \\
\text { come, si escucha algún ruido interrumpe } \\
\text { come hasta terminar el plato }\end{array}$ & $\begin{array}{l}1 \\
2 \\
3 \\
4\end{array}$ \\
\hline & alimento balanceado & $\begin{array}{l}\text { no lo come } \\
\text { come en el lugar de descanso } \\
\text { come un poco, da una vuelta por la casa } \\
\text { come hasta terminar el plato }\end{array}$ & $\begin{array}{l}1 \\
2 \\
3 \\
4\end{array}$ \\
\hline \multirow{2}{*}{ defecación / micción } & fuera del hogar & $\begin{array}{l}\text { no defeca ni orina } \\
\text { lo hace una vez cerca de la casa } \\
\text { lo hace } 2 \text { o más veces cerca de la casa } \\
\text { lo hace } 2 \text { o más veces en la plaza }\end{array}$ & $\begin{array}{l}1 \\
2 \\
3 \\
4\end{array}$ \\
\hline & dentro del hogar & $\begin{array}{l}1 \text { vez en cualquier lugar } \\
1 \text { o } 2 \text { veces en un lugar determinado } \\
1 \text { o } 2 \text { veces cerca del papel de diario } \\
\text { siempre arriba del papel de diario }\end{array}$ & $\begin{array}{l}1 \\
2 \\
3 \\
4\end{array}$ \\
\hline
\end{tabular}

Tabla 4. Comportamientos sociales registrados en las sesiones experimentales. Componentes, variables y scores definidos.

\begin{tabular}{|c|c|c|c|}
\hline $\begin{array}{l}\text { categoría del } \\
\text { comportamiento }\end{array}$ & comportamiento & descripción de las variables & score \\
\hline \multirow{12}{*}{ aproximación } & \multirow{4}{*}{$\begin{array}{l}\text { a personas } \\
\text { desconocidas }\end{array}$} & huye o escapa & 1 \\
\hline & & no se acerca & 2 \\
\hline & & se acerca sin mover la cola y solo olfatea & 3 \\
\hline & & se acerca moviendo la cola, se deja acariciar & 4 \\
\hline & \multirow{4}{*}{$\begin{array}{l}\text { a los miembros } \\
\text { de la familia }\end{array}$} & no se acerca & 1 \\
\hline & & se acerca sin mover la cola & 1 \\
\hline & & se acerca y se deja acariciar & 3 \\
\hline & & se acerca con señales de juego & 4 \\
\hline & \multirow{4}{*}{ a otros perros } & no se acerca & 1 \\
\hline & & se acerca sin mover la cola y lo olfatea & 2 \\
\hline & & se acerca y realiza contacto táctil & 3 \\
\hline & & se acerca y realiza señal de juego & 4 \\
\hline \multirow{12}{*}{ juego } & \multirow{4}{*}{ con objetos } & no juega & 1 \\
\hline & & lo olfatea 1 o 2 veces y luego lo deja & 2 \\
\hline & & juega por unos minutos y luego lo deja & 3 \\
\hline & & juega por un buen rato y busca su juguete & 4 \\
\hline & \multirow{4}{*}{$\begin{array}{l}\text { con los miembros } \\
\text { de la familia }\end{array}$} & no juega & 1 \\
\hline & & se acerca, juega y luego se acuesta & 2 \\
\hline & & juega por unos minutos & 3 \\
\hline & & invita al juego y juega por un rato & 4 \\
\hline & \multirow{4}{*}{ con otros perros } & no juega & 1 \\
\hline & & lo mira, se acerca y lo olfatea & 2 \\
\hline & & vocaliza, se acerca y lo olfatea & 3 \\
\hline & & mueve la cola, realiza contacto táctil y señal de juego varias veces & 4 \\
\hline \multirow{8}{*}{ paseos } & \multirow{4}{*}{$\begin{array}{l}\text { con los dueños } \\
\text { en un lugar } \\
\text { desconocido }\end{array}$} & no quiere salir & 1 \\
\hline & & tiembla durante el paseo y se asusta con los ruidos & 2 \\
\hline & & tiembla al principio, se asusta con algunos ruidos & 3 \\
\hline & & no tiembla y se asusta con algunos ruidos & 4 \\
\hline & \multirow{4}{*}{$\begin{array}{l}\text { con los dueños } \\
\text { en un lugar } \\
\text { conocido }\end{array}$} & le cuesta salir & 1 \\
\hline & & tiembla al principio, se asusta con algunos ruidos & 2 \\
\hline & & no tiembla y se asusta con ruidos muy fuertes & 3 \\
\hline & & no tiembla, mueve la cola y pasea relajada & 4 \\
\hline
\end{tabular}

alimento balanceado en los horarios determinados. A partir de este momento comenzó a salir a pasear, utilizándose la técnica de desensibilización a los ruidos de la calle (primero el umbral de la casa, después la vereda, hasta llegar a una plaza). Si bien los perros adoptados de criaderos exhiben una alta frecuencia de ensuciar dentro de la casa y de orinar o defecar cuando se los deja solos $^{8}$, este no fue el caso, ya que a partir de los 15 días la paciente comenzó a defecar fuera de la casa y en presencia de los dueños $(p<0,001)$. Hacia los 30 días dejó de temblar y comenzó a jugar, tanto en la casa como durante los paseos, tanto con las personas como con otros perros y con diferentes objetos (Figura 2). Durante todo este tiempo la paciente no solo evolucionó física y 
conductualmente en forma favorable, sino que además no presentó ninguno de los efectos adversos descriptos para la paroxetina. Esta clase de antidepresivos inhibe la recaptación de serotonina y produce un aumento de la neurotransmisión serotoninérgica ${ }^{11}$. El sistema serotoninérgico se localiza en el núcleo del rafe dorsal y produce inhibición de los mecanismos adaptativos de apego social ${ }^{3}$, motivo por el cual su uso está indicado en el tratamiento farmacológico de patologías como miedos, fobias, depresión, ansiedad social y agitación asociada a la depresión ${ }^{3,11}$.

A diferencia de la fluoxetina, la paroxetina tiene una vida media más corta y un mecanismo de acción más rápido con poca afinidad por los receptores muscarínicos y $\alpha 1$ adrenérgicos, por lo cual su uso presenta menos riesgos colaterales anticolinérgicos, efectos de tipo sedativo y cardiovasculares ${ }^{3}$. Si bien para este fármaco se describen efectos adversos como alteración del apetito, anorexia, vómitos, diarrea, cambios en la frecuencia urinaria, insomnio, disminución de la líbido y anafilaxia, éstos no fueron observados en el transcurso de la experiencia ${ }^{3}$. Se debe destacar que la perra recuperó el apetito y luego de dos meses entró en celo.

Los animales criados en confinamiento (refugios, cría comercial, animales de laboratorio) se enfrentan a un estrés inducido por el ambiente, a una socialización inadecuada y a otras causales psico-conductuales como el miedo. La adopción no resulta sencilla para el animal, es un proceso de aprendizaje, inserción a un nuevo ambiente, cambios en la alimentación, lugar de descanso, nuevas personas y nuevos animales, entre otros factores ${ }^{16}$. El seguimiento de este proceso debería ser controlado por el veterinario no solo desde las variables conductuales sino también desde los parámetros fisiológicos que resultan útiles para considerar el estrés inducido por tales cambios.

Si bien se considera que el miedo es una estrategia adaptativa que permite al individuo evitar o defenderse de los estímulos ofensivos o peligrosos, cuando las respuestas generadas son desproporcionadas y el individuo no vuelve a su homeostasis sensorial, el bienestar se ve afectado con modificaciones del estado de salud. Hipertensión, hiperglucemia, enfermedades infecciosas recurrentes y aumento del peristaltismo, son algunas de las alteraciones que afectan a individuos que padecen estrés patológico.

En conclusión, surge que reconocer la relación de eventos entre comportamientos como los de miedo y/o fobias con alteraciones del sistema nervioso autónomo, resulta primordial para evaluar la evolución terapéutica de los pacientes. Además, permite conocer los alcances conjuntos de las terapias farmacológica y conductual, alternativas que permitirán corregir las patologías que afectan al bienestar de los pacientes.

Agradecimiento. Al Lic. Gerardo Andino y al Laboratorio Central de Redes y Programas por la colaboración técnica prestada.

\section{REFERENCIAS}

1. Bergamasco L, Osella MC, Savarino P, Larosa G, Ozella L, Manassero M, Badino P, Odore R, Barbero R, Re G. 2010. Heart rate variability and saliva cortisol assessment in shelter dog: Human-animal interaction effects. Appl Anim Behav Sci 125: 56-68.

2. Blackwell EJ, Bodnariu A, Tyson J, Bradshaw JW, Casey RA. 2010. Rapid shaping of behaviour associated with high urinary cortisol in domestic dogs. Appl Anim Behav Sci 124: 113-120.

3. Bowen J, Heath S. 2005. Behaviours problems in small animals, Elsevier, Edinburgh, p. 73-95.

4. Casey R. 2002. Fear and stress in companion animals. In: Manual of canine and feline behavioural medicine (Horwitz DF, Mills DS, Heath S Ed.), Publ. Brit Small Anim Vet Ass, Gloucester, p. 144-153.

5. Hydbring-Sandberg E, Von Walter IW, Hoglund K, Svartberg K, Swenson L, Forkman B. 2004. Physiological reactions to fear provocation in dogs. J Endocr 180 : 439-448.

6. Kobelt AJ, Hemsworth PH, Barnet JL, Butler KL. 2003. Sources of samplings variations in saliva cortisol in dogs. Res Vet Sci 75: 157-161.

7. Kolevska J, Brunclik V, Svoboda M. 2003. Circadian rhythm of cortisol secretion in dogs of different daily activities. Acta Vet Brno 72: 599-605.

8. McMillan FD, Duffy DL, Serpell JA. 2011. Mental health of dogs formerly used as "breeding stock" in commercial breeding establishments. Appl Anim Behav Sci 135: 86-94.

9. Mills D, Zulch H. 2010. Papel del miedo y de la ansiedad en el comportamiento agresivo de los perros. Vet Focus 20: 44-49.

10. Neilson JC. 2002. Fear of places or things. In: Manual of canine and feline behavioural medicine (Horwitz DF, Mills DS, Heath S Ed.), Publ. Brit Small Anim Vet Ass, Gloucester, p. 173-180.

11. Overall K. 2010. Modificación farmacológica del comportamiento en perros y gatos. Vet Focus 20: 27-36.

12. Sanchez-Ramirez JD, Uribe-Velazquez LF. 2009. Aspectos neurobiológicos implicados en el miedo animal. Biosalud 8: 189-213

13. Ulrich-Lai Y, Herman JP. 2009. Neural regulation of endocrine and autonomic stress responses. Nat Rev 10: 397409.

14. Von Borell E, Langbein J, Despres G, Hansen S, Leterrier C, Marchant-Forde J, Marchant-Forde R, Minero M, Mohr E, Prunier A, Valance D, Veissier I. 2007. Heart rate variability as a measure of autonomic regulation of cardiac activity for assessing stress and welfare in farm animals. A review. Physiol Behav 92: 293-316.

15. Walker R, Fisher J, Neville P. 1997. The treatment of phobias in the dog. Appl Anim Behav Sci 52: 275-289.

16. Wechsler B, Lea SE. 2007. Adaptation by learning: its significance for farm animal husbandry. Appl Anim Behav Sci 108: 197-214. 